\title{
Morphological Evolution of Ag/Mica Films Grown by Pulsed Laser Deposition
}

\section{Citation}

Warrender, Jeffrey M. and Michael J. Aziz. Morphological evolution of Ag/Mica films grown by pulsed laser deposition. 2003. Materials Research Society Symposium Proceedings 749, W3.1.

\section{Published Version}

http://www.mrs.org/s_mrs/sec_subscribe.asp?CID=2572\&DID=118908\&action=detail

\section{Permanent link}

http://nrs.harvard.edu/urn-3:HUL.InstRepos:2797444

\section{Terms of Use}

This article was downloaded from Harvard University's DASH repository, and is made available under the terms and conditions applicable to Other Posted Material, as set forth at http:// nrs.harvard.edu/urn-3:HUL.InstRepos:dash.current.terms-of-use\#LAA

\section{Share Your Story}

The Harvard community has made this article openly available.

Please share how this access benefits you. Submit a story.

\section{Accessibility}




\title{
Morphological Evolution of Ag/Mica Films Grown by Pulsed Laser Deposition
}

Jeffrey M. Warrender \& Michael J. Aziz

Division of Engineering and Applied Sciences

Harvard University

\begin{abstract}
Many vapor-deposited metal-on-insulator films exhibit a morphological progression with increasing thickness consisting of several distinct stages: (1) nucleation of 3-dimensional nanocrystalline islands; (2) elongation of the islands; (3) film percolation. Here we report a study of this progression during Pulsed Laser Deposition (PLD), a technique for film deposition that differs from thermal deposition in that the depositing species arrive in short energetic bursts, leading to instantaneous deposition fluxes orders of magnitude higher than can be achieved in thermal growth. Atomic Force Microscopy reveals that advancement through this same morphological progression occurs at lower thickness in PLD films relative to films grown under comparable conditions by thermal deposition, with PLD films having lower RMS roughness at a given thickness. We also observe that for a constant amount deposited per pulse, films deposited at higher laser pulse frequency are further advanced in morphological state. Kinetic Monte Carlo simulations reveal that PLD nucleation behavior differs from that of thermally deposited films, and this can account for the observed differences. Simulations also reveal a scaling of the percolation thickness with pulse frequency that is consistent with experiment.
\end{abstract}

\section{INTRODUCTION}

Metal-on-insulator thin films typically grow according to the Volmer-Weber growth mode, in which atoms grow in $3 \mathrm{D}$ islands on the surface. ${ }^{1-5}$ As the islands grow larger, they impinge upon other islands. When this occurs, the islands may begin to coalesce, with atoms being exchanged between them, driving them to become a single island. Such is the case for liquid droplet growth. ${ }^{6}$ Metal nanocrystalline "droplets" also appear to undergo a "liquidlike" coalescence; however, rather than being much faster than deposition and surface processes, as is the case for liquid droplets, the time scale for nanocrystal coalescence is thermally activated, and thus occurs on the same time scale as deposition and surface diffusion. For low substrate temperatures, no redistribution of material whatsoever may be observed. At higher temperature, some restructuring takes place. The time scale for this process increases as the participating islands grow larger. ${ }^{7}$ As such, as discussed by Jeffers et al. ${ }^{3}$, at some point, given a system of two coalescing islands, the time for a third island to grow to impinge with one of these islands becomes less than the time for the islands to coalesce. It is at this point that clusters of coalescing islands remain elongated on the surface; they have become kinetically frozen. Further deposition joins these elongated clusters, forming a tortuous network of channels that continues to fill in with subsequent deposition. ${ }^{2}$

The extensive knowledge base on metal-on-insulator film growth by thermal deposition or Molecular Beam Epitaxy (MBE) makes it an excellent model system with which to study surface kinetics for film growth by Pulsed Laser Deposition (PLD). PLD differs from MBE in two essential ways: (1) the depositing species arrive in short bursts, on the order of 10-100 $\mu \mathrm{s}$, and (2) the depositing species have a kinetic energy of 10-100 eV, compared with about 1 
$\mathrm{eV}$ for MBE. For the purposes of this paper, we primarily consider the effect of (1), leaving (2) for future work.

We study this phenomenon using a combination of experiments and Kinetic Monte Carlo simulations. Two investigations are reported. In the first, our PLD results are compared to MBE results grown under comparable conditions, the most important of which is that the time-averaged deposition flux is the same. This corresponds to an investigation of the effect of varying the instantaneous deposition flux. In the second, the amount of material deposited during each pulse is held constant, and the laser pulse rate is varied. This corresponds to investigating the effect of varying the average deposition flux.

\section{EXPERIMENT}

Films were prepared by Pulsed Laser Deposition (PLD) in a vacuum chamber with base pressure of $2 \times 10^{-8}$ Torr. The target was $99.999 \%$ pure $\mathrm{Ag}$, and was irradiated with a $\mathrm{KrF}$ excimer laser beam (Lambda Physik LPX 305), wavelength $248 \mathrm{~nm}$. The laser spot size was $3 \mathrm{~mm} \times 1 \mathrm{~mm}$, with an energy density of $5 \mathrm{~J} / \mathrm{cm}^{2}$. The target was rotated during deposition. The target was irradiated for 2 minutes at $10 \mathrm{~Hz}$ with a shutter preventing deposition prior to each growth run. The substrate was a $2.5 \mathrm{~cm}$ square of Muscovite Mica (Alpha Aesar), freshly cleaved in deionized water, mounted $11 \mathrm{~cm}$ from the target, and heated to $200^{\circ} \mathrm{C}$. The deposition rate was $0.015 \mathrm{~nm} /$ shot, as determined by Rutherford Backscattering Spectrometry after growth. The laser was operated at 3, 10, and $30 \mathrm{~Hz}$.

Films were analyzed with ex situ Atomic Force Microscopy (AFM) and Scanning Electron Microscopy (SEM) to check morphology, and with a 2-point resistance measurement to determine film continuity.

\section{KINETIC MONTE CARLO SIMULATIONS}

Kinetic Monte Carlo (KMC) simulations were implemented. The simulation algorithm is based on code written by Erlebacher and described elsewhere. ${ }^{8}$ The substrate is a $512 \times 512$ square lattice with periodic boundary conditions. Two adjacent adatoms immediately nucleate a new island, and an atom adjacent to an island is instantly and irreversibly incorporated into the island. To reproduce the Volmer-Weber film growth geometry, islands are assumed to remain hemispherical, with a contact angle of $90^{\circ}$. This is accomplished by immediately relocating an impinging atom to the nearest vacant site to the projected center of mass of the island. This is tantamount to assuming that surface diffusion on the metal island is much faster than surface diffusion on the insulating substrate.

When two islands come into contact, they begin to coalesce. This is accomplished by implementing a procedure devised by Carrey and Maurice and described elsewhere. ${ }^{9}$ The islands are held next to each other for a time given by

$$
\tau=\frac{R^{4}}{B}
$$

where $R$ is the size of the smaller island and $B$ is an input parameter that contains materials parameters such as the surface diffusivity and the surface free energy. $B$ has dimensions of

$a^{4} / s e c$, where $a$ is the substrate lattice parameter. During this time the islands may continue to grow, and may begin to coalesce with other islands. After the time for coalescence has 
elapsed, the islands are replaced with a new island at the center of mass of the original twoisland system.

At each data-taking interval, the total number of clusters of coalescing islands, $N_{C}$, and the number of constituent islands of each cluster, $I_{C}$, is recorded. The average number of islands in a coalescing cluster is calculated. When this number exceeds 2 , according to Carrey and Maurice, further film growth tends to cause other islands to impinge with the coalescing cluster in less time than will be required for the two constituent islands to finish coalescing. As such, $I_{C}=2$, represents a transition from compact hemispherical islands to elongated islands. The thickness at which $I_{c}=2$ is defined as the elongation transition thickness $d_{T .}{ }^{9}$

\section{RESULTS AND DISCUSSION}

Effect of varying instantaneous flux at constant average flux. PLD films were grown at $3 \mathrm{~Hz}$, and these were compared with films grown at roughly the same time-averaged deposition rate by Baski and Fuchs by MBE deposition. ${ }^{2}$ The goal of our experiment was to ascertain the influence of the instantaneous deposition flux, at constant time-averaged deposition flux. The results appear in Fig. 1. As can be seen, the PLD-deposited films exhibit similar morphologies. However, the in-plane length scale is reduced for the PLD films and the film thickness at which the comparable features are observed is less for the PLD films. Additionally, the RMS roughness of the films is less for the PLD films at corresponding points in the morphological progression.

In this comparison, the PLD growth rate is about three times slower than the MBE growth rate. However, raising the growth rate to match the MBE rate is expected to reduce rather than increase feature sizes and therefore the growth rate difference should not be responsible for the observed differences. The next section illustrates this point.

$\mathrm{KMC}$ simulations were performed to compare PLD to MBE fluxes, and while our simulations don't currently permit the study of systems as large as several microns on a side, they do qualitatively exhibit similar trends. Fig. 2 shows representative images from two simulations performed at $70 \mathrm{~K}$, with a barrier to diffusion $E_{D}=0.1 \mathrm{eV}, B=500$, and average deposition flux $F_{\text {avg }}=0.25$ monolayers (ML)/s. (a) shows a continuously deposited film and (b) shows a film grown at $10 \mathrm{~Hz}$ (pulse duration $10^{-4} \mathrm{~s}$ ). The pulsed film has a higher density of islands, which are smaller than those of the continuous film. Additionally, (c) shows that the pulsed film reaches $I_{C}=2$ with less deposition than the continuously deposited film. Both of these trends are consistent with the experimental observations shown in Fig. 1.

Effect of varying average flux at constant instantaneous flux. The laser pulse rate was varied without varying any characteristics of the individual pulses. Fig. 3(a) shows AFM images for the three principal morphological stages at each of three laser pulse rates. The films grown at $30 \mathrm{~Hz}$ advanced through the characteristic progression at the lowest film thickness, followed by the $10 \mathrm{~Hz}$ and $3 \mathrm{~Hz}$ films. Furthermore, the in-plane length scale of the features is smaller at higher pulse rate. Fig. 3(b) and (c) show simulated films that have both reached percolation, both deposited at $F=0.025 \mathrm{ML} /$ pulse. Fig. 3(b) corresponds to deposition of 100 pulses at $100 \mathrm{~Hz}$; Fig. 3(c) corresponds to 176 pulses at $10 \mathrm{~Hz}$. The length scale and number of shots to percolation are lower for the $100 \mathrm{~Hz}$ film, which is consistent with the experimental observation. 


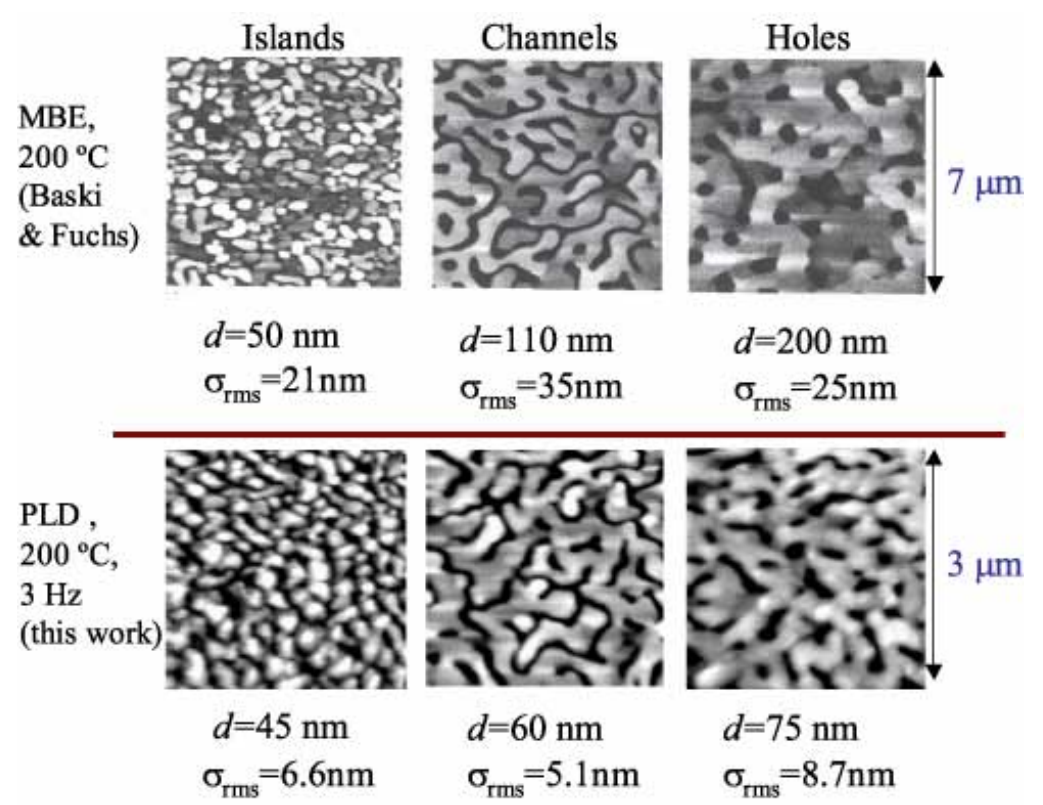

Fig. 1. Comparison of Ag(111)/mica film growth grown by $M B E^{2}$ and PLD (this work). Progression from elongated islands to channels to holes is similar, but transitions occur for thinner films in PLD than in MBE. PLD also appears to result in smaller features (note lateral length scale) and smoother films.

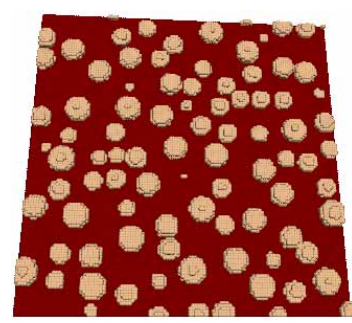

(a)

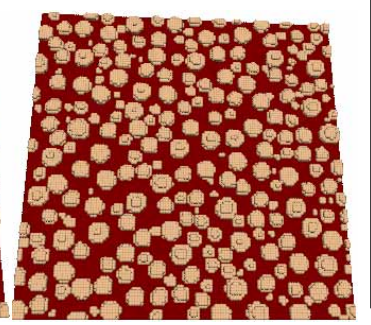

(b)

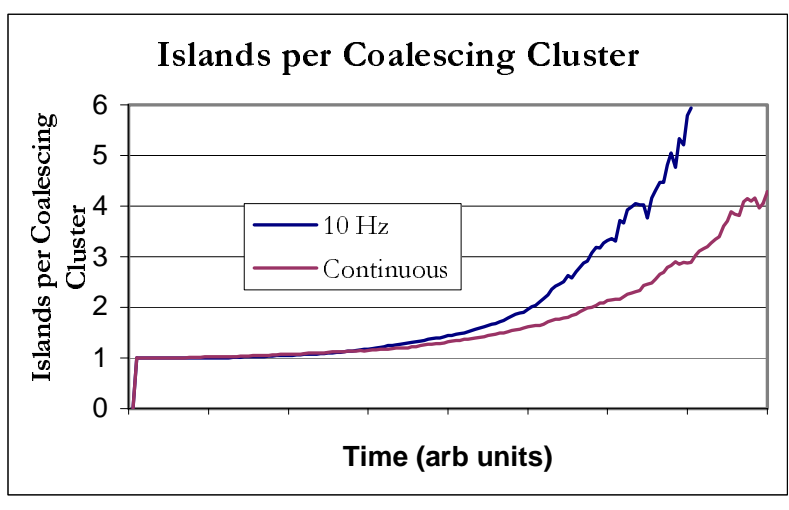

(c)

Fig. 2. KMC simulated films grown at $F_{\text {avg }}=0.25 \mathrm{ML} / \mathrm{s},($ a) continuous and $(b)$ pulsed at 10 $\mathrm{Hz}$ deposition. Note the higher density of small islands in (b). (c) The number of islands per coalescing cluster vs. time. The $10 \mathrm{~Hz}$ simulation reaches the elongation transition $\left(I_{C}=2\right)$ sooner than the continuously deposited sample.

Fig. 4a (4b) plots the film thickness at percolation (elongation transition) vs. laser pulse rate for experimental (simulated) films. Both show a monotonically increasing trend that is well fit by a power law. The simulated films all used $E_{D}=0.1 \mathrm{eV}, T=70 \mathrm{~K}, B=500, F=$ $0.025 \mathrm{ML} / \mathrm{shot}$. The exponent for the simulated case is consistent with the exponent of 0.33 predicted for continuous deposition by Jeffers et al., and by Carrey and Maurice. ${ }^{3,9}$ Interestingly, we tentatively attribute the consistency to the experimental temperature being sufficiently high that the adatoms reach islands very quickly after the flux is turned off, therefore almost all nucleation and growth occurs while the pulse is on. Thus, we simply have a case 
of continuous deposition (albeit at anomalously high deposition rate) interrupted by a time period of varying length during which no substrate diffusion or island growth occurs. Other simulations were performed at a lower temperature, $55 \mathrm{~K}$, giving a value of $E_{D} / k_{B} T=21.1$, for which free adatoms would persist on the surface until the arrival of the subsequent pulse. For that case, at least two scaling regimes of transition thickness $v s$. pulse frequency are observed, as shown in Fig. 4(c). More experimental work in the low-frequency / hightemperature regime needs to be performed to ascertain whether the experimental films are consistent with the "low $T$ " or "high $T$ " simulation results. In either case, the scaling exponents are a bit lower for the experiment than the simulation, but not dramatically so.

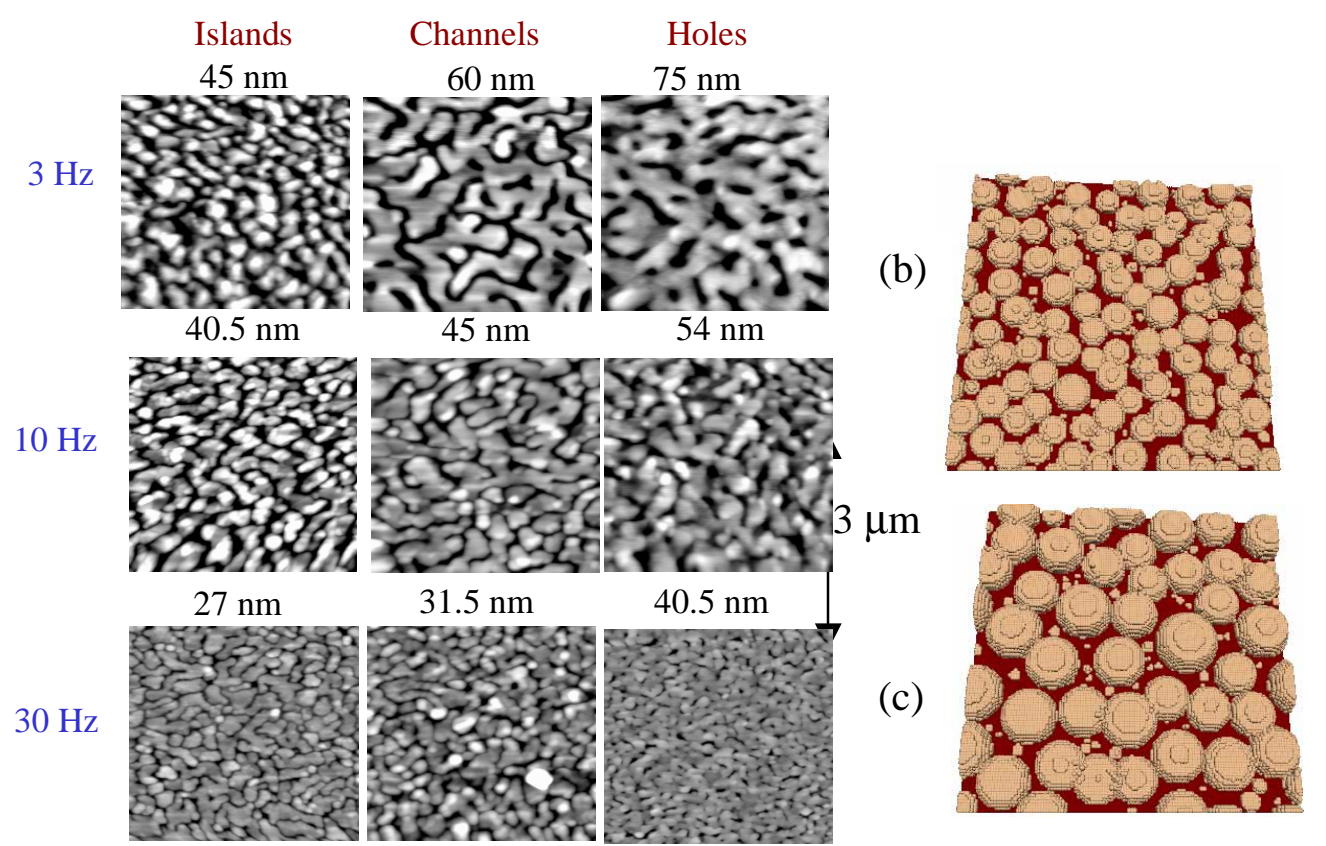

(a)

Fig. 3. (a) Film morphology vs. film thickness and pulse rate. Films deposited at higher pulse rate advance through the progression sooner. (b) and (c) $128 \times 128$ regions of simulated films grown at $F=0.025 \mathrm{ML} /$ pulse. (b) 100 pulses, $100 \mathrm{~Hz}$ (c) 176 pulses, $10 \mathrm{~Hz}$.

\section{SUMMARY}

PLD Ag films on mica grew in the morphological progression observed in MBE, changing from 3D islands to elongated islands, and finally to a percolated channel structure and subsequent hole filling. The PLD films were compared with films grown by MBE, to ascertain the effect of instantaneous deposition flux. The PLD films reached comparable morphological stages with relatively less deposited material, and with the films having features of smaller lateral size and lower RMS roughness. These observations are qualitatively consistent with KMC simulations, which show that the large difference between instantaneous flux results in a much higher nucleation density for PLD films.

PLD films grown at varying pulse rates were compared. The films that were grown at higher pulse rate were more advanced in morphological state at comparable thickness and had smaller lateral features. These results were also consistent with KMC simulations, which 


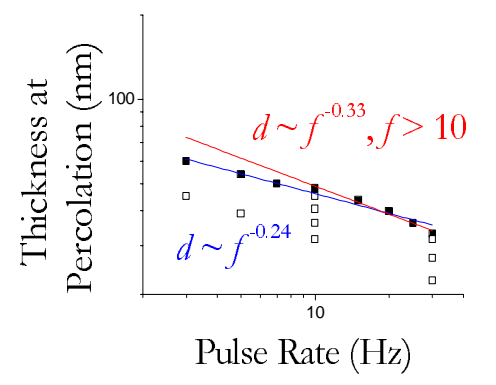

(a)

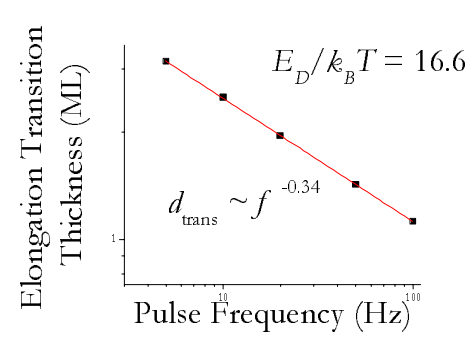

(b)

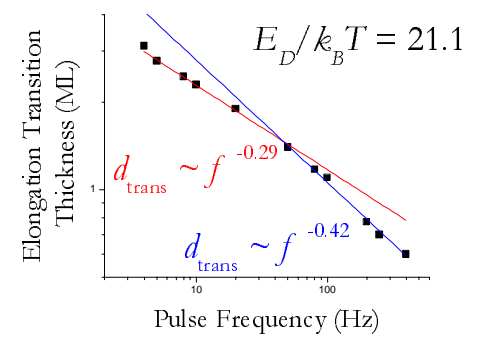

(c)

Fig. 4. (a) Thickness at which film conducts electrically vs. pulse rate. Open symbols represent films which did not conduct in a simple 2 wire continuity test; filled symbols correspond to films that did conduct. The blue line represents the best fit to the data; the red line represents the best fit to the data for frequencies $>10 \mathrm{~Hz}$. (b) and (c) Elongation transition thickness vs. pulse frequency from KMC simulations. (b) $E_{D} / k_{B} T=16.6$, for which all island nucleation and adatom diffusion occurs while the pulse is on. (c) $E_{D} / k_{B} T=21.1$, for which adatom diffusion and nucleation of new islands can occur in between pulses at high pulse frequency.

showed that for elevated temperature, all surface transport happens during the deposition pulse; thus, the main difference between pulse rates is the relative amount of time that is allowed for coalescences to complete before further island growth occurs. Simulations exhibited power law scaling of elongation transition thickness with laser pulse rate, consistent with the experimental results. Given that KMC simulations, which invoke none of the energetic aspects of PLD growth, can effectively explain the experimental results, it appears that the non-energetic characteristics of the PLD deposition flux - its enormous instantaneous magnitude and pulsed character - can adequately explain most of the morphological characteristics of Ag/Mica PLD film growth in the regime that we have studied to date.

This research was supported by NSF grant DMR-972736 and DOE grant DE-FG02$01 \mathrm{ER} 45947$.

1 D. W. Pashley, M. H. Jacobs, M. J. Stowell, et al., Philosophical Magazine 10, 127 (1964).

A. A. Baski and H. Fuchs, Surface Science 313, 275 (1994).

G. Jeffers, M. A. Dubson, and P. M. Duxbury, Journal of Applied Physics 75, 5016 (1994).

4 X. Yu, P. M. Duxbury, G. Jeffers, et al., Physical Review B (Condensed Matter) 44, 13163 (1991).

P. Brault, A.-L. Thomann, and C. Andreazza-Vignolle, Surface Science 406, L597 (1998).

$6 \quad$ B. J. Briscoe and K. P. Galvin, Physical Review A 43, 1906 (1991).

7 F. A. Nichols and W. W. Mullins, Journal of Applied Physics 36, 1826 (1965).

8 J. D. Erlebacher, in Dynamics of Crystal Surfaces and Interfaces, edited by P. M. Duxbury and T. Pence (Plenum, New York, 1997).

9 J. Carrey and J.-L. Maurice, Physical Review B (Condensed Matter and Materials Physics) 63, 245408/1 (2001). 